\title{
Erratum to: Imaging findings of fibrolamellar hepatocellular carcinomas on ultrasonography: A comparison with conventional hepatocellular carcinomas
}

Tiantian $\mathrm{Fu}^{\mathrm{a}}$, Hong Ding ${ }^{\mathrm{b}, *}$, Chen $\mathrm{Xu}^{\mathrm{c}}$, Yuli Zhu ${ }^{\mathrm{a}}$, Liyun Xue ${ }^{\mathrm{a}}$ and Feng Lin ${ }^{\mathrm{d}, *}$

${ }^{a}$ Department of Ultrasound, Shanghai Institute of Medical Imaging, Zhongshan Hospital, Fudan University, Shanghai, China

${ }^{\mathrm{b}}$ Department of Ultrasound, Huashan Hospital, Fudan University, Shanghai, China

${ }^{\mathrm{c}}$ Department of Pathology, Zhongshan Hospital, Fudan University, Shanghai, China

${ }^{\mathrm{d}}$ School of Computer Science and Engineering, Nanyang Technological University, Singapore,

Singapore

[Clinical Hemorheology and Microcirculation 77 (1) (2021), 49-60] http://doi.org/10.3233/CH-200896

The affiliation of the corresponding author Hong Ding was incomplete. The correct affiliation should also include "a Department of Ultrasound, Shanghai Institute of Medical Imaging, Zhongshan Hospital, Fudan University, Shanghai, China”.

The affiliations should be corrected as follows. Changes are marked by underlines:

Tiantian $\mathrm{Fu}^{\mathrm{a}}$, Hong Ding ${ }^{\mathrm{a}}, \mathrm{b}, *$, Chen $\mathrm{Xu}^{\mathrm{c}}$, Yuli Zhu ${ }^{\mathrm{a}}$, Liyun Xue ${ }^{\mathrm{a}}$ and Feng Lin ${ }^{\mathrm{d}, *}$

${ }^{a}$ Department of Ultrasound, Shanghai Institute of Medical Imaging, Zhongshan Hospital, Fudan University, Shanghai, China

${ }^{\mathrm{b}}$ Department of Ultrasound, Huashan Hospital, Fudan University, Shanghai, China

${ }^{\mathrm{c}}$ Department of Pathology, Zhongshan Hospital, Fudan University, Shanghai, China

${ }^{\mathrm{d}}$ School of Computer Science and Engineering, Nanyang Technological University, Singapore, Singapore

The corrected footnote should read:

*Corresponding authors: Hong Ding, MD, PhD, Department of Ultrasound, Huashan Hospital, Fudan University, No. 12 Middle Urumqi Road, Shanghai 200040, China; Department of Ultrasound, Shanghai Institute of Medical Imaging, Zhongshan Hospital, Fudan University, Shanghai, China. Tel.:+86 1365188 6013; E-mail: ding hong@fudan.edu.cn; Feng Lin, Professor, School of Computer Science and Engineering, Nanyang Technological University, Singapore 639798, Singapore. E-mail: ASFLIN @ ntu.edu.sg. 